\title{
Study of Aetiological and Endoscopic Profile of Patients with Upper Gastrointestinal Bleed in North East India - A Hospital Based Cross Sectional Study
}

\author{
H.R. Bharath ${ }^{1}$, Anupam Dutta², Ajit Kumar Pegu ${ }^{3}$, Balaji Dhanabalan4, Bhabani Sankar Dhal ${ }^{5}$ \\ 1, 2, 3, 4, 5 Department of Medicine, Assam Medical College and Hospital, Dibrugarh, Assam, India.
}

\section{ABSTRACT}

\section{BACKGROUND}

Upper Gastro-Intestinal Bleeding (UGIB) is one of the common complaints with which patients present to casualty. It is associated with significant morbidity and mortality. The aetiological spectrum of UGIB is variable in different geographical regions. Our study aimed to analyse the aetiology, endoscopic profile, mortality, Rockall score and predictors of mortality in patients with UGIB, in North East India.

\section{METHODS}

This cross-sectional study was conducted at Assam Medical College and Hospital in North East India. We enrolled patients with age 12 years and above, who were admitted between July 2019 and January 2020 with a history suggestive of UGIB. Demographic data of the patients was collected, after which they underwent clinical examination, and upper GI endoscopy. Mean \pm standard deviation was used to express continuous variables. Frequency and percentage were used to express categorical variables. Test of significance for qualitative data was assessed by Chisquare test (for $2 \times 2$ tables). $\mathrm{P}$ value less than 0.05 was taken as statistically significant.

\section{RESULTS}

We analysed 117 patients diagnosed with UGIB [80.34 \% male, $19.60 \%$ female], ratio of male to female of [4.08:1] was seen. The most common symptom was melena 87 patients (74.15\%), endoscopy finding showed that $48.71 \%$ had oesophageal and / or gastric varices, $26.49 \%$ had peptic ulcers, $17.94 \%$ had gastric erosions / duodenal erosions / erosive gastritis, $1.7 \%$ had Mallory-Weiss tear, $1.7 \%$ had gastric malignancy, $1.7 \%$ had GJ stoma bleed, $1.7 \%$ had both oesophageal varices and peptic ulcer disease. Partial gastric outlet obstruction was observed in peptic ulcer disease in 2 patients ( $6.45 \%$ of total peptic ulcer disease patients). $73.75 \%$ patients had Rockall score $<5$ and $26.49 \%$ patients had Rockall score $>6$. H. pylori infection (assessed by RUT) was an independent predictor of upper GI bleed in both variceal and non-variceal bleed $[p<0.001]$. The mortality in our study was $7.69 \%$. Predictors of mortality in the study population were, patients with variceal bleed $[p=<0.001]$, Rockall score $>6[p=0.013]$, and chronic liver disease $[p<0.001]$. The average duration of hospital admission of the study population is about $4.6+/-0.4$ days.

\section{CONCLUSIONS}

The study reported oesophageal varices was the most common cause of UGIB, followed by peptic ulcer in North East India. H. pylori was an independent predictor of both variceal and non-variceal bleed. Partial gastric outlet obstruction (GOO) was one of the common benign complication of peptic ulcer disease. Variceal bleed, Rockall score $>6$, chronic liver disease were predictors of mortality.

\section{KEY WORDS}

Oesophageal Varices, Upper Gastrointestinal Bleeding, Upper GI Endoscopy, Variceal Bleed
Corresponding Author:

Dr. Bharath HR,

3rd Year Postgraduate Trainee,

Department of Medicine, Assam Medical

College and Hosital, Dibrugarh, Assam,

India.

E-mail: bharathhramesh@gmail.com

DOI: $10.14260 / \mathrm{jemds} / 2020 / 683$

How to Cite This Article:

Bharath HR, Dutta A, Pegu A, et al. Study of aetiological and endoscopic profile of the patients with upper gastrointestinal bleed in North East India - a hospital based cross sectional study. J Evolution Med Dent Sci 2020;9(42):3112-3116, 10.14260/jemds/2020/683

Submission 25-04-2020,

Peer Review 12-09-2020,

Acceptance 18-09-2020,

Published 19-10-2020.

Copyright (c) 2020 Bharath H.R. et al. This is an open access article distributed under Creative Commons Attribution License [Attribution 4.0 International (CC BY 4.0)] 


\section{BACKGROUND}

Acute gastrointestinal [GI] bleeding is one of the lifethreatening GI emergencies. It is one of the common causes for hospitalizations worldwide. Upper GI bleeding is defined as bleeding from a source proximal to the ligament of Treitz, it is associated with a varied range of clinical severity, such as from insignificant bleeds to catastrophic severe haemorrhage. ${ }^{1}$ The incidence of acute upper GI bleed is in between 50 to 100 per 100,000 persons per year, ${ }^{2}$ about $70 \%$ of acute upper GI bleed episodes occur in patients with more than 60 years $^{3}$ and the incidence may be directly proportional to age, because of the greater intake of nonsteroidal anti-inflammatory drugs [NSAIDs] in elderly, which provoke the genesis of ulcer.

Helicobacter pylori infection is associated with increased peptic ulcer disease and upper GI bleed risk. ${ }^{4}$ Helicobacter pylori infection can be diagnosed easily by Rapid urease test [RUT] with almost $95 \%$ sensitivity and $100 \%$ specificity. ${ }^{5}$ Comorbid diseases are associated with increased incidence with non- variceal upper GI bleed. 6

Patients can be divided as having either variceal or nonvariceal sources of upper GI haemorrhage as the two have different management protocols and prognosis. ${ }^{7}$ Variceal source includes lesions that arise by virtue of portal hypertension, namely gastro-oesophageal varices and portal hypertensive gastropathy; and the non-variceal includes peptic ulcer, erosive gastritis, reflux esophagitis, MalloryWeiss tear, gastric tumours, etc.

GOO is one of the complications of PUD (Peptic Ulcer Disease) and can present with early satiety, bloating, weight loss, indigestion, nausea and vomiting. On clinical examination, epigastric fullness and succession splash may be heard due to trapped air and fluid in the stomach. Ulcers that manifest with GOO are often located in the pyloric channel or duodenal bulb. ${ }^{8}$

Mortality rates ranges in between $12 \%$ to $35 \%$ in patients with age more than 60 years, in contrast with $<10 \%$ for patients with age less than 60 years, ${ }^{9}$ and overall mortality rate is between of $5 \%-11 \%$.

The primary diagnostic test for upper GI bleed workup is endoscopy. Sensitivity of $92 \%-98 \%$ and specificity of $30 \%$ $100 \%$ is present for endoscopy for the diagnosis of upper GI bleed.10 Severity scoring system like Rockall's score, helps to identify the patients who are at higher risk for rebleed and mortality. ${ }^{11}$

At present, there are limited data on clinical and endoscopic profile of patients of upper GI bleed from North East India, hence this study was planned to identify clinical and endoscopic profile of patients with upper GI bleed and to study the mortality rates and its association with Rockall scoring.

\section{METHODS}

This is a cross-sectional study, which was carried out in the Department of Medicine at Assam Medical College and Hospital, Dibrugarh, in North East India. We enrolled patients from inpatient setting in this study. A total of 117 patients with upper GI bleed, presented to Assam Medical College and
Hospital, between July 2019 and January 2020 and underwent upper GI endoscopy. UGI bleed presents as a hematemesis i.e. vomitus of red blood or coffee ground material and or the passage of melena i.e. black tarry stools, ${ }^{12}$ reported by the patient or the patient's family members or witnessed by medical staff.

\section{Ethics}

The study was approved by the Ethics Committee of Assam Medical College and Hospital, Dibrugarh. We obtained a written informed consent from all study participants before enrolling them in the study.

\section{Inclusion Criteria}

Patients admitted with UGI bleed less than 5 days with age more than 12 years.

\section{Exclusion Criteria}

Presence of contraindication for endoscopy i.e. unconscious patient, orodental abnormalities, recent MI ( $<3$ months), acute pulmonary oedema, suspicion of perforated viscus, and patient not willing for consent.

We elicited information about risk factors habits such as alcohol consumption, consumption of anti-inflammatory drugs (NSAIDS), antiplatelet drugs and steroids to all study participants and serological tests for hepatitis B, hepatitis $C$ and serum electrolytes to selected study participants. Rockall scoring was done to all patients included in the study.13

All the study participants received the standard line of management for upper GI bleed. Collected data was analysed and results were given as numbers and percentages.

\section{Statistical Analysis}

Data was entered into computer Microsoft Excel and exported to SPSS version 20 for analysis. Continuous variables were expressed as mean \pm standard deviation. Categorical variables were expressed as frequency and percentage. Chi-square test (for $2 \times 2$ tables) was used as test of significance for qualitative data. $\mathrm{P}$ value was considered statistically significant when it was less than 0.05 .

\section{RESULTS}

The study population comprised of 117 patients with upper GI bleed who came to Assam Medical College and Hospital from July 2019 to January 2020 . The patients in the study were with age above 12 years of age, and the eldest patient was 80 years old and youngest being 17 years. The study population's mean age was $54+/-14$ years. The study population comprised of 94 males (80.34\% patients) and 23 females (19.60\% patients), and male to female ratio is 4.08:1. The presenting symptoms of the patients included in the study are as follows, 
it was seen that the majority of the patients had melena 87 patients $(74.15 \%)$, hematemesis 14 patients $(11.96 \%)$, both hematemesis and melena 16 patients $(13.67 \%)$.

\begin{tabular}{|cc|}
\hline Age & Frequency (Percentage) \\
13 - 25 Years & $3(2.56 \%)$ \\
$25-40$ Years & $17(14.56 \%)$ \\
$40-65$ Years & $64(54.70 \%)$ \\
$65-80$ Years & $33(28.20 \%)$ \\
Mean age & $54+/-14$ years \\
Gender & Frequency (Percentage) \\
Male & $94(80.34 \%)$ \\
Female & $23(19.60 \%)$ \\
Male : Female ratio & $4.08: 1$ \\
\hline Table 1. Showing Demographic Profile
\end{tabular}

\begin{tabular}{|cc|}
\hline Symptoms & Frequency (Percentage) \\
Melena & $87(74.15 \%)$ \\
Hematemesis & $14(11.96 \%)$ \\
Both & $16(13.67 \%)$ \\
\hline \multicolumn{2}{|c|}{ Table 2. Clinical Profile } \\
\hline
\end{tabular}

History of chronic alcohol intake was noted in 95 patients $(81.91 \%)$ and drug history was depicted, about 7 patients (5.98\%) were on NSAIDS, 3 patients $(2.5 \%)$ had both chronic alcohol and NSAIDS intake, 5 patients ( $4.27 \%$ ) were on antiplatelet drugs, 2 patients $(1.7 \%)$ were on steroids and 5 patients (4.27 \%) didn't had any drug history or chronic alcohol intake.

Chronic alcoholic intake patients had a risk for both variceal and non- variceal, no difference between both groups. [p = 0.1]. Among 117 patients, the commonest co-morbidity was chronic liver disease seen in 47 patients (40.17\%), followed by hypertension seen in 29 patients $(24.2 \%), 21$ patients $(17.94 \%)$ had diabetes mellitus, 5 patients $(4.27 \%)$ had ischemic heart disease, 3 patients (2.56 \%) had chronic kidney disease. Chronic liver disease is the strong predictor of variceal bleed. [ $\mathrm{p}=<0.001]$.

\begin{tabular}{|cc|}
\hline Risk Factors & Frequency (Percentage) \\
Chronic Alcohol Consumption & $95(81.91 \%)$ \\
NSAIDs & $7(5.98 \%)$ \\
Both Chronic Alcohol Consumption and NSAIDs & $3(2.5 \%)$ \\
Antiplatelet Drugs & $5(4.27 \%)$ \\
Steroids & $2(1.7 \%)$ \\
Comorbidities & Frequency (Percentage) \\
Chronic Liver Disease & $47(40.17 \%)$ \\
Hypertension & $29(24.2 \%)$ \\
Type 2 Diabetes Mellitus & $21(17.94 \%)$ \\
Ischemic Heart Disease & $5(4.27 \%)$ \\
Chronic Kidney Disease & $3(2.56 \%)$ \\
UGI Endoscopy & Frequency (Percentage) \\
Variceal Cause- & \\
Oesophageal and / Gastric Varices & $57(48.71 \%)$ \\
Non-Variceal Cause- & \\
Peptic Ulcer Disease & $31(26.49 \%)$ \\
Gastritis) & $21(17.94 \%)$ \\
Erosions (Gastric / Duodenal / Erosive & $2(1.7 \%)$ \\
Mallory Weis Tear & $2(1.7 \%)$ \\
Gastric Malignancy & $2(1.7 \%)$ \\
GJ Stoma Bleed & $2(1.7 \%)$ \\
Both Variceal and Non-Variceal Cause- & \\
\hline Table 3. Risk Factors, Comorbidity Profile and Endoscopic Profile
\end{tabular}

The upper GI endoscopy profile of the study population were as follows, it was seen that, 57 patients $(48.71 \%)$ had gastro oesophageal varices, 21 patients (17.94 \%) had gastric erosion / duodenal erosions / erosive gastritis, 31 patients (26.49\%) had peptic ulcer (gastric and duodenal ulcers), 2 patients $(1.70 \%)$ had gastric malignancy, 2 patients $(1.70 \%)$ had Mallory- Weiss tear, 2 patients (1.70\%) had GJ stoma bleed and 2 patients (1.70\%) had both variceal and nonvariceal (peptic ulcer) upper GI bleed.
In our study 7 patients (12.28 \% of variceal bleed patients) had hepatic encephalopathy, showing the influence of upper GI bleed on other organs.

\begin{tabular}{|cc|}
\hline $\begin{array}{c}\text { Aetiology Data of Variceal Upper GI Bleed } \\
\text { (n = 57) }\end{array}$ & $\begin{array}{c}\text { Frequency } \\
\text { (Percentage) }\end{array}$ \\
Alcohol Liver Disease (ALD) & $41(71.92 \%)$ \\
Chronic Hepatitis B & $5(8.77 \%)$ \\
Chronic Hepatitis C & $3(5.26 \%)$ \\
Cryptogenic & $4(7.01 \%)$ \\
Non-Cirrhotic Portal Fibrosis (NCPF) & $4(7.01 \%)$ \\
H. Pylori Infection (RUT) & Frequency (Percentage) \\
RUT positive overall & $65(55.55 \%)$ \\
RUT positive out of Non-Variceal cases ( $\mathrm{n}=60)$ & $53(88.33 \%)$ \\
RUT positive out of Variceal cases (n = 57) & $22(38.59 \%)$ \\
\hline Table 4. Aetiological Profile of Variceal Upper GI Bleed and \\
Relationship of H. pylori Infection with Upper GI Bleed \\
\hline
\end{tabular}

The helicobacter pylori infection association with upper GI bleed was tested using RUT (Rapid Urease Test) and overall positive RUT was in 65 patients (55.55\%). Positive RUT associated with non- variceal upper GI bleed (mainly Peptic ulcer disease, duodenal / gastric erosions and erosive gastritis) was seen in 53 patients $(88.33 \%$ of total nonvariceal upper GI bleed) and positive RUT associated with variceal upper GI bleed was seen in 22 patients $(38.59 \%$ of total variceal upper GI bleed). $H$. pylori infection is an independent predictor of both variceal and non-variceal bleed [p $=<0.001]$.

Partial gastric outlet obstruction was observed in peptic ulcer disease in 2 patients $(6.45 \%$ of total peptic ulcer disease patients) both clinically and by upper GI endoscopy. The mortality rate in the study population was $7.69 \%$ (9 patients). All the patients who expired had variceal cause and none had non- Variceal cause. Rockall score was done for the study population, 86 patients ( $73.75 \%$ ) had Rockall score $<5$ and 31 patients $(26.25 \%)$ had Rockall score $>6.9$ patients $(7.69 \%)$ expired overall. Risk factors for death was assessed such as comorbidities like chronic liver disease in 9 patients $(100 \%)$, Type 2 diabetes mellitus in 3 patients (33.33\%), hypertension in 2 patients (22.22\%), chronic kidney disease in 2 patients $(22.22 \%)$. Variceal bleed $[p=0.06]$, Rockall score $>6[p=$ $0.013]$ and chronic liver disease $[p=0.001]$ were strong predictors of mortality.

\begin{tabular}{|cc|}
\hline Mortality Rate & Frequency (Percentage) \\
Overall Mortality Rate & $9(7.69 \%)$ \\
Variceal Cause Mortality Rate & $9(15.78 \%)$ \\
Non-Variceal Cause Mortality Rate & $0(0 \%)$ \\
& Frequency (Percentage) \\
Comorbidity Profile of Expired Patients (n = 9) & $9(100 \%)$ \\
Rockall Score $>6$ & $9(100 \%)$ \\
Chronic Liver Disease & $3(33.33 \%)$ \\
Type 2 Diabetes Mellitus & $2(22.22 \%)$ \\
Hypertension & $2(22.22 \%)$ \\
Chronic Kidney Disease & No of days \\
Hospital Stay & $4+/-0.4$ days \\
Overall Average Hospital Stay & 4.3 days \\
Variceal Cause Hospital Stay & 3.7 days \\
Non-Variceal Cause Hospital Stay & \\
Table 5. Mortality Rate, and Comorbidity Profile of \\
Expired Patients and Duration of Hospital Stay
\end{tabular}

\begin{tabular}{|ccc|}
\hline Rockall Score Details & Rockall Score < \5 & Rockall Score > / 6 \\
Variceal Bleed (57) & $36(63.15 \%)$ & $21(36.85 \%)$ \\
Non-Variceal Bleed (60) & $50(83.33 \%)$ & $10(16.67 \%)$ \\
Total 86 (73.5 \%) & $\mathbf{3 1}(\mathbf{2 6 . 4 9} \%)$ \\
\hline Table 6. Association of Rockall Score with Type of UGI Bleed \\
\hline
\end{tabular}

The average days of hospital stay in the study population is about $4+/-0.4$ days, the patients with variceal upper GI 
bleed has an average of $4.3+/-0.4$ days of hospital stay and for non- variceal upper GI bleed has 3.7 + / - 0.4 days.

\section{DISCUSSION}

Our cross-sectional study aimed to determine the aetiological and endoscopic profile of upper GI bleed, in patients with age more than 12 years, admitted in Assam Medical College and Hospital with a history of hematemesis and / or melena.

In our study, patients were between the age group of 17 to 80 years. The mean age of patients in our study was $54+/-14$ years and incidence of upper GI bleed was higher in male patients (male : female ratio 4.08:1), both of which is similar to the study done by Kashyap et al. ${ }^{14}$ In our study, out of a total of 117 patients, the majority of the study population presented with melena i.e. 87 patients $(68.11 \%)$, while 14 patients $(20.95 \%)$ with hematemesis, and 16 patients $(13.67 \%)$ with both melena and hematemesis, but the study done by Jyoti Jain et $\mathrm{al}^{15}$ showed that combination of both symptoms was the most common, followed by melena and hematemesis respectively.

Chronic alcohol consumption, use of NSAIDs, antiplatelet drugs, steroids and comorbidities are well known risk factors associated with upper GI bleed. In our study, 95 patients (81.19\%) were chronic alcohol consumer's, 7 patients (5.98 $\%$ ) had history of NSAIDs intake, 3 patients with both chronic alcohol intake and NSAIDs intake, 5 patients $(4.27 \%)$ had antiplatelet intake, 2 patients $(1.7 \%)$ with oral steroid intake.

History of intake of the above-mentioned medications are the good predictors of non- variceal bleed, since all of them had non-variceal bleed [ $p<0.001]$. Chronic alcoholic intake patients had a risk for both variceal and non- variceal, no difference between both groups [p=0.1].

Chronic liver disease was the commonest co-morbidity (40.17\%), followed by hypertension (24.2\%), diabetes mellitus (17.34\%), chronic kidney disease (2.4\%) similar to study done by Colin John Crooks et al. ${ }^{6}$

In the present study, 57 patients ( $48.71 \%$ ) had upper GI bleed due to portal hypertension related causes i.e. oesophageal varices, gastric varices and 31 patients $(26.49 \%)$ due to peptic ulcer disease, 21 patients (17.94\%) due to gastric erosion / duodenal erosions / erosive gastritis, 2 patients $(1.7 \%)$ with gastric malignancy, 2 patients $(1.7 \%)$ with Mallory- Weiss tear, 2 patients (1.7\%) with GJ stoma bleed, 2 patients $(1.7 \%)$ with both variceal and non- variceal upper GI bleed, which is similar to the study done by Jyoti Jain et al. ${ }^{15}$ But the study done by Dr Ardhendu Kumar Sen et al ${ }^{16}$ showed that peptic ulcer disease was the commonest cause of upper GI bleed. The higher number of patients with variceal bleed in our study was seen because of high prevalence of chronic alcohol consumer's (81.97\%) and chronic liver disease $(40.17 \%)$ and since ours is the only tertiary care hospital in upper Assam, majority of variceal bleed patients are supposed to be critical, all the critical patients are referred to our hospital, which may have added on to the percentage of patients with variceal bleed.

Aetiology of the variceal upper GI bleed was analysed, the most common cause was alcoholic liver disease seen in 41 patients (63.15\%), followed by chronic hepatitis B seen in 5 patients $(8.77 \%)$, cryptogenic in 4 patients $(7.01 \%)$, non- cirrhotic portal fibrosis (NCPF) in 4 patients $(7.01 \%)$ and chronic hepatitis $\mathrm{C}$ in 3 patients (5.26\%). The results are almost similar to the study done by Pranav Mahajan et al. ${ }^{7}$

Chronic liver disease was the strong predictor of variceal bleed [ $p=<0.001]$. Upper GI bleed has influence on other organs as well, such as it is one of the commonest precipitant factors in patients with decompensated liver cirrhosis to cause hepatic encephalopathy. In our study 7 patients $(12.28 \%$ of variceal bleed patients) had hepatic encephalopathy, results similar to the study done by Dr. Ajit Pegu et al. ${ }^{17}$

The rapid urease test (RUT) performed showed that overall positive RUT was in 65 patients (55.55\%). Positive RUT in non-variceal upper GI bleed (mainly peptic ulcer disease and erosive gastritis) was seen in 53 patients $(88.33 \%$ of total non- variceal upper GI bleed) which is similar to study done by Henrickkson AE et al, ${ }^{6}$ and positive RUT in variceal upper GI bleed was seen in 12 patients $(21.05 \%$ of total variceal upper GI bleed), which is similar to study done by Mohamed A. Elsebaey et $\mathrm{al}^{18}$ in which it was found to have $H$. pylori infection as high as $57.9 \%$ in patients with gastric varices and found to be an independent risk factor for bleeding in patients with gastric varices as well. Thus, H. pylori infection was an independent predictor of both variceal and nonvariceal bleed $[\mathrm{p}=<0.001]$.

GOO was observed in peptic ulcer disease in 2 patients (6.45\% of total peptic ulcer disease patients) which is the benign complication of peptic ulcer disease, according to the study done by Vivek Sukumar et al ${ }^{19}$ showed that benign cause of GOO was about $38.60 \%$ patients, in which peptic ulcer disease was the most common cause and benign causes can be managed conservatively majority of the times. Overall inhospital mortality in our study was $7.69 \%$ (9 patients). All the patients who expired had variceal upper GI bleed and none had non-variceal upper GI bleed. The mortality rate is high for variceal bleed, almost similar to the study done by Hysanita Jaka et al.20

Rockall score done for the study population shows that 86 patients (73.75\%) had Rockall score $<5$ and 31 patients $(26.25 \%)$ had Rockall score $>6.9$ patients $(7.69 \%)$ expired overall. Details of the expired patients were assessed, such as comorbidities like chronic liver disease in 9 patients (100\%), Type 2 diabetes mellitus in 3 patients (33.33\%), hypertension in 2 patients $(22.22 \%)$, chronic kidney disease in 2 patients $(22.22 \%)$ and all 9 patients $(100 \%)$ had Rockall score $>6$, which is also similar to the study done Dewan et al. ${ }^{12}$ But variceal bleed $[\mathrm{p}=0.06]$, Rockall score $>6[\mathrm{p}=0.013]$ and chronic liver disease $[\mathrm{p}=0.001]$ were strong predictors of mortality.

The average days of hospital stay before discharge in the study population is about $4+/-0.4$ days, the patients with variceal upper GI bleed has an average of $4.3+/-0.4$ days of hospital stay and for non- variceal upper GI bleed has $3.7+$ / 0.4 days. The average days of hospital stay is less compared to the study done by Dewan et al.11

\section{CONCLUSIONS}

This cross-sectional study showed that variceal bleed was the commonest cause and melena the commonest presentation of upper GI bleed in North East India. NSAIDs intake was the major independent risk factor for non-variceal bleed. Chronic 
alcohol consumption and $H$. pylori infection were independent risk factors for both variceal and non-variceal UGIB. UGI endoscopy is an important modality in both diagnosis and therapy in upper GI bleed. Alcoholic liver disease was the commonest aetiology of variceal bleed. Partial GOO is one of the common benign complication of peptic ulcer disease. Variceal bleed, Rockall score $>6$ and chronic liver disease were strong predictors of mortality.

Data sharing statement provided by the authors is available with the full text of this article at jemds.com.

Financial or other competing interests: None.

Disclosure forms provided by the authors are available with the full text of this article at jemds.com.

We would like to thank Dr. S. Hazarika, Dr. Chayanika Dutta, Dr. Arijit Das, Dr. Ramanan BBV, Dr. Sanchu Sreeraj, from Medical Unit - 5 , Assam Medical College and Hospital, for their help during the study.

\section{REFERENCES}

[1] Rockall TA, Logan RF, Devlin HB, et al. Selection of patients for early discharge or outpatient care after acute upper gastrointestinal haemorrhage. National Audit of acute upper gastrointestinal haemorrhage. Lancet 1996;347(9009):1138-40.

[2] El-Tawil AM. Trends on gastrointestinal bleeding and mortality: where are we standing? World J Gastroenterol 2012;18(11):1154-8.

[3] Hreinsson JP, Kalaitzakis E, Gudmundsson S, et al. Upper gastrointestinal bleeding: incidence, etiology and outcomes in a population - based setting. Scand J Gastroenterol 2013;48(4):439-47.

[4] Khalifehgholi M, Shamsipour F, Ajhdarkosh H, et al. Comparison of five diagnostic methods for helicobacter pylori. Iron J Microbiol 2013;5(4):396-401.

[5] Henriksson AE, Edman AC, Nilsson I, et al. Helicobacter pylori and the relation to other risk factors in patients with acute bleeding peptic ulcer. Scand J Gastroenterol 1998;33(10):1030-3.

[6] Crooks CJ, West J, Card TR. Comorbidities affect risk of nonvariceal upper gastrointestinal bleeding. Gastroenterology 2013;144(7):1384-93.

[7] Mahajan P, Chandail VS. Etiological and endoscopic profile of middle aged and elderly patients with upper gastrointestinal bleeding in a tertiary care hospital in North India: a retrospective analysis. J Midlife Health 2017;8(3):137-141.
[8] Ashwin K, Visruja RL. A clinical study of gastric outlet obstruction. Int Surg J 2018;5(12):3913-8.

[9] Christensen S, Riis A, Nørgaard M, et al. Short-term mortality after perforated or bleeding peptic ulcer among elderly patients: a population- based cohort study. BMC Geriatr 2007;7:8.

[10] Kim BSM, Li BT, Engel A, et al. Diagnosis of gastrointestinal bleeding: a practical guide for clinicians. World J Gastrointest Pathophysiol 2014;5(4):467-78.

[11] Dewan KR, Patowary BS, Bhattarai S. A study of clinical and endoscopic profile of acute upper, gastrointestinal bleeding. Kathmandu Univ Med J (KUMJ) 2014;12(45):215.

[12] Laine L. Gastrointestinal bleeding. In: Jameson LJ, Kasper LD, Longo LD, et al. eds. Harrison's Principles of internal medicine. $20^{\text {th }}$ edn. New York: Mcgraw Hill Education 2018:272-6.

[13] Rockall TA, Logan RF, Devlin HB, et al. Risk assessment after acute upper gastrointestinal haemorrhage. Gut 1996;38(3):316-21.

[14] Kashyap R, Mahajan S, Sharma B, et al. A clinical profile of acute upper gastrointestinal bleeding at moderate altitude. JIACM 2005;6(3):224-8.

[15] Jain J, Rawool A, Banait S, et al. Clinical and endoscopic profile of the patients with upper gastrointestinal bleeding in central rural India: a hospital- based cross- sectional study. J Mahatma Gandhi Inst Med Sci 2018;23(1):13-8.

[16] Sen AK, Goswami P, Neog P, et al. An etiological study of upper gastro-intestinal bleeding with special reference to helicobacter pylori infection in peptic ulcer disease. JMSCR 2016;4(8):11696-9.

[17] Pegu AK, Dutta A, Ray A, et al. A clinical study of precipitating factors of overt hepatic encephalopathy in decompensated cirrhosis from North East India. IOSRJDMS 2016;15(6):51-3.

[18] Elsebaey MA, Tawfik MA, Elshweikh SA, et al. Impact of helicobacter pylori infection on gastric variceal bleeding among patients with liver cirrhosis. Gastroenterol Res Pract 2019;2019:6529420.

[19] Sukumar V, Ravindran C, Prasad RV. Demographic and etiological patterns of gastric outlet obstruction in Kerala, South India. N Am J Med Sci 2015;7(9):403-6.

[20] Jaka H, Koy M, Liwa A, et al. A fibreoptic endoscopic study of upper gastrointestinal bleeding at Bugando Medical Centre in North Western Tanzania: a retrospective review of 240 cases. BMC Res Notes 2012;5:200. 